10.1117/2.1201512.006250

\title{
Using quantum effects in nanomaterials for unique identification
}

Robert J. Young, Jonathan Roberts, and Phillip Speed

Current-voltage measurements of atomic-scale variations in resonant tunneling diodes can be used to provide secure fingerprints.

Authentication and identification are critical to information security systems. Traditionally, these processes are achieved with the use of secret keys that are stored in electronic memories, or with difficult-to-clone systems (e.g., fingerprints or holograms). The persistent development of technology, however, means that the barrier to cloning such systems is becoming lower. Moreover, counterfeiting, device spoofing, and identity fraud are formidable problems in all markets. The ideal solution, therefore, would be to produce a nano-fingerprint from the atomic arrangement of a structure embedded within a device. By shrinking down to the atomic scale, the challenge of cloning the system becomes as difficult as possible, i.e., the density of secure information is maximized and the number of resources required to read the fingerprint is minimized.

Physically unclonable functions (PUFs) are information security devices that capture the value of deriving an identity from a physical attribute of an object. ${ }^{1}$ For example, in a so-called optical $\mathrm{PUF}^{1}$ an interference pattern is derived by passing coherent light through an object that contains an array of imperfections. These structures are easy to fabricate and they are difficult to reproduce, because of randomness in the fabrication processes. There are problems, however, associated with modern PUFs. ${ }^{2}$ With the advance of manufacturing techniques, cloning PUFs is becoming easier because they are based on classical properties. In addition, significant resources are often required to measure PUFs. They can also be emulated and are susceptible to sophisticated attacks.

In our work, we solve these PUF problems by shrinking the systems down to the nanoscale. At this scale, the effects of quantum confinement become crucial to the properties of the device. We have shown that it is possible to extract unique

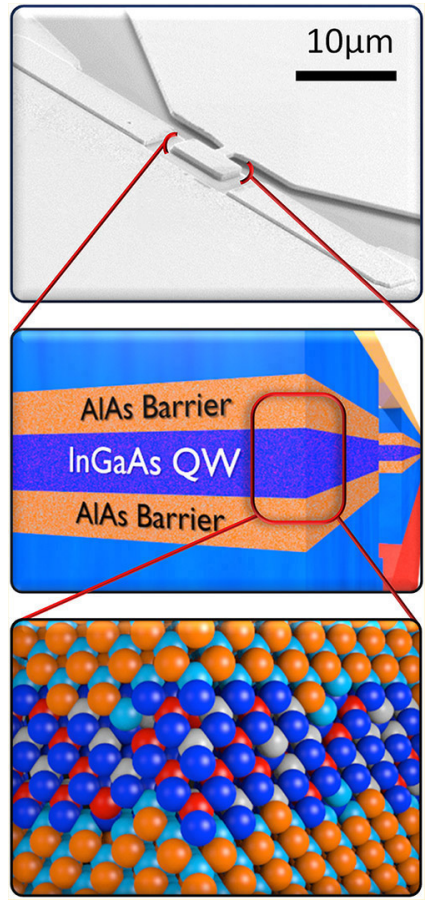

Figure 1. Scanning electron microscope image (top) of a typical resonant tunneling diode, which is used to produce an electronic spectrum from the nanomaterial (middle) within it. The quantum well (QW) region of the nanomaterial is highlighted (bottom) to illustrate the randomness of its structure. AlAs: Aluminum arsenide. InGaAs: Indium gallium arsenide.

signatures from nanostructures embedded within our quantum confinement PUFs (QC-PUFs). ${ }^{3}$ Our simple electronic components are compatible with traditional semiconductor fabrication processes, which makes them easy to integrate. In addition, the security of our QC-PUFs is based on atomic-scale structures, so they are extremely difficult to clone. We also store the 
input-output behavior of the QC-PUFs in a database, which allows us to provide track-and-trace capability for any tagged device.

Our QC-PUFs are made possible by the behavior of electrons in semiconductors when the material's dimensions are reduced to the nanoscale, i.e., the electrons become sensitive to atomic composition. ${ }^{4}$ When electrons interact with a material that has a diameter on the order of its de Broglie wavelength, the electrons experience quantum confinement. As such, the energy spectrum of the electrons becomes discrete, with values that are highly dependent on the width and composition of the material. We have produced and characterized simple devices, ${ }^{3}$ known as resonant

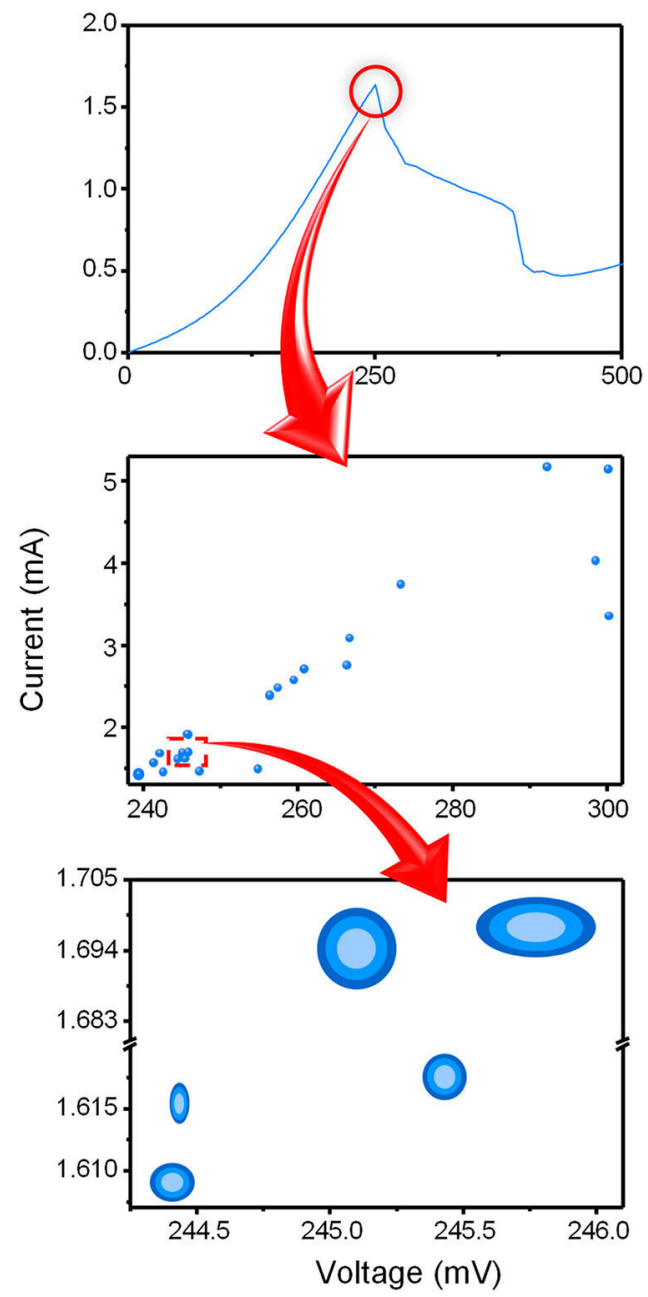

Figure 2. Current-voltage measurements from resonant tunneling diodes. Top: The spectrum from a single device. Middle: Comparison of current peak positions from 26 diodes. Bottom: Zoomed-in region that highlights the confidence levels (95, 99.9, and 99.997\%) associated with the measurements. tunneling diodes, ${ }^{5}$ to read these unique energy spectra. These devices (see Figure 1) consist of tunnel barriers, i.e., layers that inhibit electron flow. These barriers surround a quantum well, which is the nanomaterial from which a fingerprint is derived. When a voltage is applied to the device, the current depends on the energy of the confined levels within the well. Therefore, when we sweep the voltage over a range of values, we produce a spectrum that is unique to the nanomaterial. In this spectrum, the current peaks when the electron flow through the device is maximized. This occurs when there is a resonance with the quantum-confined energy levels in the well.

We have assessed the potential of our resonant tunneling diodes by testing 26 of the devices. These devices were selected from a large array that had been manufactured in parallel with the use of standard semiconductor fabrication processes. We performed the simplest fingerprinting operation with each of the structures, i.e., we fit the first resonant current peak that was found when we applied an increasing voltage to the devices. The results of this operation (see Figure 2) show that each device produced a unique result. The confidence bands shown in Figure 2 were produced by repeating the measurement of each device several times.

From the data shown in Figure 2, we predict that our quantum well resonant tunneling structure can be used to provide on the order of $10^{3}$ unique identities. ${ }^{3}$ This significantly exceeds the previous record for identities per unit volume achieved from a PUF, ${ }^{3}$ but it is far from the limit of this new technology. The degree of uniqueness extracted from each device could be increased (by orders of magnitude) by considering other spectral features of the measurement. Alternatively, multiple diodes could be connected in series, programmatically, to produce longer unique identities (with a bit depth that scales exponentially with physical size). There is also the ability to 'reset' the physical structure and fingerprint of a given resonant tunneling diode. This can be achieved through over-biasing if the security of the device is ever compromised. This feature is unique, among PUF technologies, to resonant tunneling diodes.

In this work we have extracted unique identities from atomic-scale variations of a nanomaterial within a simple semiconductor diode. These fingerprints could be used to prevent fraud and counterfeiting in a wide range of markets. The fundamental scale of our devices maximizes the difficulty of cloning, and their semiconductor nature makes them easy to massproduce and integrate. We are currently exploring an optical analog to the devices. This development would allow remote reading of atomic-scale identities from nanomaterials in a 
surface coating. The optical properties of quantum dots, for example, are highly dependent on their atomic makeup. ${ }^{6}$ We are also currently developing commercial applications of these new security technologies through our spin-off company Quantum Base. $^{7}$

Robert J. Young is supported by the Royal Society through a University Research Fellowship (grant UF110555). Jonathan Roberts is supported by the Engineering and Physical Sciences Research Council 'Nownano' Doctoral Training Centre (grant EP/L01548X/1).

\section{Author Information}

\section{Robert J. Young and Jonathan Roberts}

Physics Department

Lancaster University

Lancaster, United Kingdom

Robert J. Young is a Royal Society Research Fellow. He leads a quantum information and security group and is a co-founder of Quantum Base.

Jonathan Roberts is studying for a $\mathrm{PhD}$ as part of the Engineering and Physical Sciences Research Council-funded doctoral training center 'Nownano.' He is jointly supervised by Robert Young and Max Migliorato at the University of Manchester.

\section{Phillip Speed}

Quantum Base Ltd

Lancaster, United Kingdom

Phillip Speed is a co-founder of Quantum Base. He has more than 25 years of experience in the information and communications technology industry.

\section{References}

1. R. Pappu, B. Recht, J. Taylor, and N. Gershenfeld, Physical one-way functions, Science 297, pp. 2026-2030, 2002

2. C. Helfmeier, C. Boit, D. Nedospasov, and J.-P. Seifert, Cloning physically unclonable functions, IEEE Int'1 Symp. Hardware-Oriented Secur. Trust, pp. 1-6, 2013 doi:10.1109/HST.2013/6581556

3. J. Roberts, I. E. Bagci, M. A. M. Zawawi, J. Sexton, N. Hulbert, Y. J. Noori, M.

P. Young, et al., Using quantum confinement to uniquely identify devices, Sci. Rep. 5, p. 16456, 2015. doi:10.1038/srep16456

4. http://www.nobelprize.org/nobel_prizes/physics/laureates/2000/The Nobel Prize in Physics 2000. Accessed 25 November 2015.

5. L. V. Iogansen, The possibility of resonance transmission of electrons in crystals through a system of barriers, J. Experim. Theoret. Phys. 18, pp. 146-150, 1964.

6. S. Tomić, J. Pal, M. A. Migliorato, R. J. Young, and N. Vukmirović, Visible spectrum quantum light sources based on $I n_{x} G a_{1-x} N / G a N$ quantum dots, ACS Photon. 2, pp. 958-963, 2015 .

7. http://quantumbase.com/Quantum Base website. Accessed 4 December 2015. 